\title{
Prostate Cancer-Secreted Exosomal miR-1275 Modulates Osteoblast Proliferation and Differentiation by Targeting SIRT2/RUNX2 Cascade
}

\section{Zihao Zou (D 1256249626@qq.com )}

Third Affiliated Hospital of Guangzhou Medical College

Ranran Dai

Third Affiliated Hospital of Guangzhou Medical College

Nan Deng

Third Affiliated Hospital of Guangzhou Medical College

Wei Su

Southern Medical University

Ping Liu

Third Affiliated Hospital of Guangzhou Medical College

\section{Research}

Keywords: prostate cancer, exosomes, miR-1275, SIRT2, osteoblast differentiation

Posted Date: September 18th, 2020

DOI: https://doi.org/10.21203/rs.3.rs-78100/v1

License: (c) (1) This work is licensed under a Creative Commons Attribution 4.0 International License. Read Full License 


\section{Abstract}

\section{Background}

Cancer-derived exosomes can promote tumor metastasis by delivering miRNAs from cancer cells to recipient cells. miR-1275 is suggested to be an important regulator involved in prostate cancer (PCa) metastasis; however, the association of miR-1275 with the microenvironment of PCa bone metastasis is unclear.

Materials and methods

The level of relative genes and proteins were detected by qPCR and western-blot, cell ability were examined by CCK-8, the identification of target gene was analyzed by dual-luciferase reporter assay,exosomes were isolated from the PC3-derived conditioned medium by ultracentrifugation.

Result

we found that miR-1275 could be transferred from PCa cells to osteoblasts via exosomes. Exosomal miR1275 significantly accelerated the proliferation of osteoblasts and the expression levels of osteoblastspecific genes, such as osteocalcin (OCN), type I collagen (COL-1), and osteopontin (OPN). Moreover, exosomal miR-1275 increased the expression of RUNX2, a master modulator of osteoblast differentiation, by down-regulation of SIRT2, which in turn influenced the proliferation and differentiation of osteoblasts.

Conclusions

Our findings indicate that exosomal miR-1275 is a promoter of osteoblast activity, which may contribute to PCa bone metastasis.

\section{Introduction}

Prostate cancer ( $\mathrm{PCa}$ ) was the second most frequently diagnosed cancer and the fifth leading cause of cancer mortality among men worldwide in 2012 [1]. The incidence of PCa is increasing worldwide in recent years. Each year 1.6 million men are diagnosed with PCa and 366,000 men die of this disease [2]. Bone metastasis is a major cause of the mortality of PCa patients, which requires the communication between PCa cells and the metastatic microenvironment [3,4]. Therefore, understanding the molecular mechanism underlying the interaction between PCa cells and osteoblasts will be crucial for developing novel therapeutic strategies against metastatic $\mathrm{PCa}$.

Exosomes are small extracellular vesicles, which are involved in multiple cellular processes, such as tumor initiation, growth, progression, metastasis, drug resistance, and immune escape [5]. Exosomes contains many genetic material, proteins, and lipids and act as potential signaling molecules between cancer cells and the surrounding cells [6]. Exosomes also carry different nucleic acids, such as microRNAs (miRNAs) [7]. miRNAs are a group of small (19 22 nt) endogenous non-coding nucleotides 
that modulate cell growth, differentiation, and metabolism through posttranscriptional regulation of gene expression [7]. It has been reported that cancer cells deliver their exosomes comprising miRNAs to the metastatic site and modify the microenvironment, thus facilitating cancer metastasis [8]. The intercommunication between different cells via exosomal miRNAs is suggested to be an important way of interaction between tumor cells and the tumor microenvironment [9]. Multiple studies have been demonstrated that miR-1275 plays an important role in cancer progression [10-12]. Using the whole miRNome analysis, Nam et al. have shown that miR-1275 is a potential candidate associated with PCa metastasis [13]. However, the function of miR-1275 involved in the microenvironment of PCa bone metastasis remains unclear.

In this study, we found that miR-1275 could be released from PCa cells and transferred to osteoblasts. Treatment with exosomes from miR-1275-overexpressing PCa cells (miR-1275-exo) significantly increased the activity of osteoblasts, whereas treatment with miR-1275 inhibitor abolished this effect. Moreover, our data demonstrated that miR-1275-exo exerted its function by regulating (Sirtuin 2) SIRT2/Runt-related transcription factor 2 (RUNX2) cascade. Our findings revealed an enhancing effect of exosomal miR-1275 on osteoblast activity, which may contribute to osteoblastic metastasis of PCa.

\section{Methods}

\subsection{Cell culture}

Human PC3 prostate cancer cells and hFOB1.19 osteoblasts were obtained from ATCC (USA). PC3 cells were cultured in RPMI1640 medium and hFOB1.19 cells were culture in DMEM/F12 medium (HyClone, USA). All media were supplemented with $100 \mathrm{U} / \mathrm{ml}$ penicillin, $100 \mu \mathrm{g} / \mathrm{ml}$ streptomycin (Gibco, USA), and $10 \%$ FBS (Gibco, USA). The cells were maintained at $37^{\circ} \mathrm{C}$ in a humidified atmosphere with $5 \% \mathrm{CO} 2$.

\subsection{Exosome isolation}

Exosomes were isolated from the PC3-derived conditioned medium by ultracentrifugation. Conditioned medium was collected from $48 \mathrm{~h}$ cell cultures, followed by centrifugation at $500 \mathrm{~g}$ for 20 min and 40 minutes at $10,000 \mathrm{~g}$. After filtration with a $0.2 \mu \mathrm{m}$ sterilized filter (Millipore), the supernatants were ultracentrifuged at $100,000 \mathrm{~g}$ for $70 \mathrm{~min}$ at $4{ }^{\circ} \mathrm{C}$. The exosomes were washed once with PBS, followed by a second ultracentrifuged at $100,000 \mathrm{~g}$ for $70 \mathrm{~min}$ at $4^{\circ} \mathrm{C}$. The exosomes were resuspended in PBS finally. The size distribution of the exosomes was observed on a TEM-1400plus transmission electron microscope (TEM). The concentration of exosomes was determined using a BCA protein assay kit (Thermo Fisher, USA).

\subsection{Exosome uptake assay}

Exosomes were labeled using the PKH67 Green Fluorescent Cell Linker Kit according to the manufacturer's instructions. In brief, $100 \mu \mathrm{l}$ exosomes isolated from PC3 cells with different treatments were diluted in $1 \mathrm{ml}$ diluent $\mathrm{C}$ from the kit and $6 \mu \mathrm{PKH} 26$ dye was diluted in $1 \mathrm{ml}$ diluent $\mathrm{C}$. The dilutions 
were mixed continuously for $30 \mathrm{~s}$ and kept at room temperature for $5 \mathrm{~min}$. Then $2 \mathrm{ml} 10 \%$ BSA in PBS was added to quench the staining. Labeled exosomes were washed in $10 \mathrm{ml}$ PBS and collected by centrifugation at $100,000 \mathrm{~g}$ for $70 \mathrm{~min}$. hFOB1.19 cells were then incubated with exosomes for $24 \mathrm{~h}$. After washing with PBS, cells were fixed with $4 \%$ paraformaldehyde for 10 minutes. Nuclei were stained with DAPI solution. Images were captured by a confocal laser scanning microscope LSM410 (Carl Zeiss, Germany)

\subsection{Real-time PCR}

Total RNA from cells was isolated using TRIzol reagent (Life Technologies, USA) and total RNA from exosomes was extracted using the mirVana miRNA Isolation Kit (Ambion, USA). The RNA was reverse transcribed into first-strand cDNA using the High Capacity cDNA Reverse Transcription Kit (Thermo Fisher, USA) according to the manufacturer's instructions. qRT-PCR was performed using the Power SYBR Green Master Mix (Roche) and conducted on an ABI 7500 (Applied Biosystems, USA). The amplification conditions were as follows: $95^{\circ} \mathrm{C}$ for $10 \mathrm{~min}$, followed by 40 cycles of $95^{\circ} \mathrm{C}$ for $15 \mathrm{~s}$ and $60^{\circ} \mathrm{C}$ for $45 \mathrm{~s}$. GAPDH or $\mathrm{U} 6$ were used as the internal controls for mRNA and miRNA, respectively. The relative expression was calculated using $2^{-\triangle \triangle C T}$ method. The primers used for qRT-PCR were as follows: miR-1275 forward, 5'-CTCTGTGAGAAAGGGTGTGG-3' and miR-1275 reverse, 5'-TCTGCCTTGGGAAAATAAG-3'; U6 forward, 5'-GCTTCGGCAGCACATATACTAAA-3' and U6 reverse, 5'-GCTTCACGAATTTGCGTGTCAT-3'; OCN forward: 5'-GCCCTCACACTCCTCGCCCTATT-3' and OCN reverse: 5'-GGGTCTCTTCACTACCTCGCTGCC-3'; OPN forward: 5'-ACAGCATCGTCGGGACCAGACTCGT-3', OPN reverse: 5'-

GGTAGTGAGTTTTCCTTGGTCGGCG-3'; COL-I forward, 5'-CAGCCGCTTCACCTACAGC-3' and COL-I reverse: 5'-TTTTGTATTCAATCACTGTCTTGCC-3' Runx2 forward: 5'-CCCCTCCTACCTGAGCCAGATGACG-3' and Runx2 reverse: 5'-AAGGGCCCAGTTCTGAAGCACCTGA-3'; GAPDH forward: 5'-

ATGGAAATCCCATCACCATCT-3' and GAPDH reverse: 5'-GGTTGAGCACAGGGTACTTTATT-3'.

\subsection{Luciferase reporter assay}

The wild-type 3'-UTR and the 3'-UTR mutant that contains the mutated miR-1275 binding site of SIRT2 were amplified by PCR and inserted into the pGL3-control vector (Promega, USA) to construct luciferase reporter vectors. 293T cells were seeded in 24-well plates and were co-transfected with the firefly luciferase reporter vector and the Renilla luciferase control vector with miR-1275 mimics or control mimics by Lipofectamine 2000 (Invitrogen, USA). $24 \mathrm{~h}$ post-transfection, the firefly and Renilla luciferase activities were detected using a Dual-Luciferase Reporter Assay System (Promega, USA). The firefly luciferase activity was normalized to Renilla luciferase activity.

\subsection{Western blotting}

Cells were washed once with PBS and lyzed using RIPA lysis buffer (CWBIO, China). The protein concentration was measured using the BCA method (Thermo Fisher, USA). The protein samples were separated by SDS-polyacrylamide gel electrophoresis and transferred to PDVF membrane. The membrane was blocked with $5 \%$ non-fat milk and incubated with anti- antibodies overnight at $4{ }^{\circ} \mathrm{C}$. After 
washing three times with TBST, the membrane was incubated with HRP-coupled second antibody. The protein bands were detected by Immobilon Western kit (Millipore, USA).

\subsection{Statistical analysis}

All data were presented as the mean \pm SD. Statistical analyses were performed with SPSS 19.0 (SPSS Inc. USA). Analysis between two groups were assessed by two-tailed Student's t-test while comparisons of more than two groups were performed with one-way multivariate analysis of variance (ANOVA) followed by Turkey's test. A $P$-value $<0.05$ was considered statistically significant.

\section{Results}

\subsection{Characterization of exosomes from PCa cells}

PC3 cells cultured in the exosome-free medium were transfected with miR-1275 or control miRNA and exosomes were isolated from the culture medium by centrifugation. The morphology of the isolated exosomes was examined by TEM. As shown in Figure 1A, exosomes from PC3 cells overexpressing miR1275 (miR-1275-exo) or control miRNA (NC-exo) exhibited spherical morphology with a diameter range of $50 \sim 100 \mathrm{~nm}$. To further characterize the isolated exosomes, the expression levels of exosomal markers TSG-101 and CD63 were detected by Western blot. Both NC-exo and miR-1275-exo were positive for TSG101 and CD63 (Fig. 1B). We then determined the expression of miR-1275 in exosomes using qRT-PCR. The expression of miR-1275 in miR-1275-exo was significantly higher than that in No-exo (Fig. 1C).

\section{$3.2 \mathrm{miR}-1275$ is transferred to osteoblasts via exosomes}

miRNAs can be transferred from cells to cells via exosomes to execute their function [14]. To address whether miR-1275 could be transferred from PCa cells to osteoblasts via exosomes, we labeled miR1275-exo and NC-exo with PKH67 (green), followed by incubation with osteoblasts. After $72 \mathrm{~h}$ of incubation, PKH67 lipid dye was observed in the osteoblasts (Fig. 2A), indicating a transfer of exosomes to osteoblasts. Treatment with miR-1275-exo resulted in a remarkable increase in miR-1275 expression in osteoblasts, while treatment with NC-exo had no significant effect on miR-1275 level (Fig. 2B).

\subsection{Exosomal miR-1275 enhances osteoblast proliferation and differentiation}

Next, we determined whether exosomal miR-1275 could affect the proliferation of osteoblasts. CCK-8 assay showed that treatment with miR-1275-exo significantly increased the viability of osteoblasts compared with NC-exo (Fig. 3A). Transfection with miR-1275 mimics further extended the enhancement in osteoblast viability induced by miR-375-exo. On the contrary, treatment with miR-1275 inhibitor blocked the effect of miR-375-exo (Fig. 3A). In addition, the mRNA and protein levels of osteogenesis-related genes, such as osteocalcin (OCN), type I collagen (COL-1), and osteopontin (OPN) were higher in miR1275-exo group than in NC-exo group, as characterized by qRT-PCR, Western blot, and immunofluoresence staining (Figure 3B-D). Treatment with miR-1275 mimics further increased the expression of OCN, COL-1, and OPN induced by miR-1275-exo, whereas transfection with miR-1275 
inhibitor abolished the increase induced by miR-1275-exo (Figure 3B-D). Together, these results suggest that exosomal miR-1275 has a positive effect on osteoblast activity.

\subsection{SIRT2 is a target of miR-1275}

To explore how miR-1275 modulates osteoblast activity, we search the potential downstream targets of miR-1275 by mRNA target-predicting algorithms (TargetScan and miRDB). Among the potential targets, SIRT2 was overlapped and chosen as the candidate target (Fig. 4A). To confirm that SIRT2 is a target of miR-1275, luciferase report assay was performed. The activity of wild-type 3'-UTR of SIRT2 was significantly decreased after overexpressing miR-1275, while there was no significant change in the activity of the mutated 3'-UTR of SIRT2 (Fig. 4B). Transfection of miR-1275 markedly repressed the expression of SIRT2 (Fig. 4C). Consistently, treatment with miR-1275-exo also resulted in a downregulation of SIRT2 (Fig. 4D). Overexpression of SIRT2 restored SIRT2 expression and restrained the viability of osteoblasts increased by miR-1275-exo (Fig. 4E-F). Collectively, these results indicated that SIRT2 is a target of miR-1275.

\subsection{Exosomal miR-1275 enhances osteoblast activity via SIRT2/RUNX2 cascade}

RUNX2 is a transcription factor that plays an important role in osteoblast differentiation via modulation of osteoblast genes. We found that RUNX2 could positively regulate OCN expression (Fig. S1), which was consistent with previous studies $[15,16]$. We then investigated whether SIRT2 could affect RUNX2 expression. Western blot analysis showed that overexpression of SIRT2 suppressed the protein level of RUNX2, whereas siRNA-mediated silencing of SIRT2 elevated the expression of RUNX2 (Fig. 5A). Moreover, we found that the activity of RUNX2 promoter was decreased after overexpression of SIRT2 (Fig. 5B). In agreement with this result, the mRNA level of RUNX2 was suppressed upon SIRT2 overexpression and increased following SIRT2 knockdown (Fig. 5C). Treatment with miR-1275-exo decreased SIRT2 expression but increased RUNX2 expression and co-treatment with miR-1275 inhibitor abolished these effects (Fig. 5D). Overexpression of SIRT2 abrogated the elevation in RUNX2 expression induced by miR-1275-exo (Fig. 5E). Furthermore, knockdown of RUNX2 counteracted the increased expression of OCN, as well as the viability of osteoblasts induced by miR-1275-exo (Fig. 5F-G). Taken together, these data indicate that exosomal miR-1275 accelerates osteoblast activity through the regulation of SIRT2/RUNX2 cascade.

\section{Discussion}

$\mathrm{PCa}$ is a public health threat worldwide and a leading cause of cancer-related death in men. Communication between PCa cells and osteoblasts is important for bone metastasis of PCa; but the underlying mechanism remains unclear. In the present study, we found that PC3 cells could transferred miR-1275 to osteoblasts via exosomes. Exosomal miR-1275 had a positive effect on the proliferation and differentiation of osteoblasts. 
Exosomes are important vesicles involved in the information exchange between cells and play important roles in the occurrence, development, treatment, and prognosis of various diseases [17]. miRNAs can be transferred from cancer cells to the recipient cells to facilitate tumorigenesis and metastasis [18]. For example, hepatoma cell-secreted exosomal miR-103 can be delivered into endothelial cells and increase vascular permeability, resulting in enhanced metastasis [19]. Exosomal miR-105 can be transferred from breast cancer cells to cancer-associated fibroblasts and thus promotes tumor growth [20]. Colorectal cancer (CRC)-derived miR-25-3p can be delivered to endothelial cells via exosomes and promotes vascular permeability and angiogenesis, consequently enhances CRC metastasis [21]. In this study, we found that miR-1275 could be delivered to osteoblasts via exosomes and modulated osteoblast activity.

miR-1275 has been reported to play a critical role in cancer development. For instance, it has been shown that miR-1275 regulates the proliferation and invasion of lung cancer cells by downregulation of LZTS3 [10]. Liu et al. have shown that miR-1275 accelerates the proliferation and migration of head and neck squamous cell carcinoma cells [11]. miR-1275 modulates the proliferation of nasopharyngeal cancer cells by targeting HOXB5 [12]. However, the function of miR-1275 in prostate cancer remains unknown. Disruption of the dynamic balance between osteoblasts and osteoclasts could lead to PCa bone metastasis. It has been reported that exosomal miR-141 increased the activity of osteogenesis cells and thus promotes PCa bone metastasis [22]. Our data showed that PCa-secreted exosomal miR-1275 significantly enhanced the activity of osteoblasts, which may contribute to PCa bone metastasis.

SIRT2 is an NAD+-dependent deacetylase that plays a crucial role in a variety of processes, such as cell proliferation, inflammation, immunity, and tumorigenesis [6]. It has been reported that increased SIRT2 expression associates with decreased TNM stage and longer survival in patients with breast cancer [23]. Mutation of SIRT2 in human cancers leads to a loss of its deacetylase activity and impaired genomic stability in cancer cells [24]. In adverse prostate cancer, decreased SIRT2 expression is correlated with worse clinicopathologic outcomes [25] and maybe responsible for the tumor occurrence and progression of PCa [26]. Here, we showed that SIRT2 is a target of miR-1275 and exosomal miR-1275 increased osteoblast activity by downregulation of SIRT2.

RUNX2 is a key transcription factor controlling the proliferation and differentiation of osteoblasts [27]. It has been reported that the deletion of RUNX2 in osteoblasts disrupts postnatal bone formation [28]. Deficiency of Runx2 resulted in an absence of osteoblasts and bone in mice [29]. Ectopic expression of Runx2 is effective for transdifferentiation of preadipocytes into fully differentiated bone-forming osteoblasts [16]. Overexpression of Runx2 enhances osteoblastic differentiation by altering the expression of osteoblastic genes, such as OCN [15]. In the present study, we found that RUNX2 was a downstream target of SIRT2 and exosomal miR-1275 upregulation RUNX2 by decreasing SIRT2 expression. Moreover, we confirmed that exosomal miR-1275 modulated osteoblast activity by the modulation SIRT2/RUNX2 cascade.

\section{Conclusion}


In summary, our data demonstrated that PCa-derived exosomal miR-1275 significantly increased the proliferation and differentiation of osteoblasts, which was mediated by the regulation of SIRT2/RUNX2 pathway. Therefore, exosomal miR-1275 is an important enhancer of osteoblast activity. Further studies are required to clarify the function of exosomal miR-1275 in PCa metastasis.

\section{Abbreviations}

OCN osteocalcin

COL-1 type I collagen

OPN osteopontin

TEM transmission electron microscope

RUNX2 Runt-related transcription factor 2

\section{Declarations}

\section{Acknowledgements}

Not applicable

\section{Ethics approval and consent to participate}

No

\section{Consent for publication}

All authors agree to be published

Funding: This work was supported by the natural science foundation of the Guangdong province (2019A1515011439)

\section{Availability of data and materials}

For data availability, please contact the corresponding author.

\section{Competing interests}

The authors declare that they have no competing interests.

\section{Authors' contributions}

Zihao Zou developed the overall research plan and oversaw the study. The manuscript is the original work of all authors and the final manuscript has been read and approved by all authors. The authors 
accept full responsibility for the design and conduct of the study, have access to the data, and controlled the decision to publish. The manuscript has not been previously published and is not being considered for publication elsewhere in whole or part in any language. None of the authors has any conflicts of interest related to the content of the manuscript.

\section{References}

1. Kimura T, Egawa S. Epidemiology of prostate cancer in Asian countries. Int J Uro/ 2018; 25:524-531.

2. Pernar $\mathrm{CH}$, Ebot EM, Wilson KM, Mucci LA. The Epidemiology of Prostate Cancer. Cold Spring Harb Perspect Med 2018; 8.

3. Kelly SP, Anderson WF, Rosenberg PS, Cook MB. Past, Current, and Future Incidence Rates and Burden of Metastatic Prostate Cancer in the United States. Eur Urol Focus 2018; 4:121-127.

4. Smith S, Wolanski P. Metastatic prostate cancer incidence in Australia after amendment to prostatespecific antigen screening guidelines. ANZ J Surg 2018; 88:E589-E593.

5. Li X, Wang Y, Wang Q, Liu Y, Bao W, Wu S. Exosomes in cancer: Small transporters with big functions. Cancer Lett 2018; 435:55-65.

6. Wang T, Xu Z, Lu Y, Shi J, Liu W, Zhang C, et al. Recent Progress on the Discovery of Sirt2 Inhibitors for the Treatment of Various Cancers. Curr Top Med Chem 2019; 19:1051-1058.

7. Yu X, Odenthal M, Fries JW. Exosomes as miRNA Carriers: Formation-Function-Future. Int J Mol Sci 2016; 17.

8. Feng W, Dean DC, Hornicek FJ, Shi H, Duan Z. Exosomes promote pre-metastatic niche formation in ovarian cancer. Mol Cancer 2019; 18:124.

9. Sun Z, Shi K, Yang S, Liu J, Zhou Q, Wang G, et al. Effect of exosomal miRNA on cancer biology and clinical applications. Mol Cancer 2018; 17:147.

10. He J, Yu L, Wang CM, Zhou XF. MiR-1275 promotes non-small cell lung cancer cell proliferation and metastasis by regulating LZTS3 expression. Eur Rev Med Pharmacol Sci 2018; 22:2680-2687.

11. Liu MD, Wu H, Wang S, Pang P, Jin S, Sun CF, et al. MiR-1275 promotes cell migration, invasion and proliferation in squamous cell carcinoma of head and neck via up-regulating IGF-1R and CCR7. Gene 2018; 646:1-7.

12. Sun KY, Peng T, Chen Z, Huang J, Zhou XH. MicroRNA-1275 suppresses cell growth, and retards G1/S transition in human nasopharyngeal carcinoma by down-regulation of HOXB5. J Cell Commun Signal 2016; 10:305-314.

13. Nam RK, Wallis CJD, Amemiya Y, Benatar T, Seth A. Identification of a Novel MicroRNA Panel Associated with Metastasis Following Radical Prostatectomy for Prostate Cancer. Anticancer Res 2018; 38:5027-5034.

14. Fang JH, Zhang ZJ, Shang LR, Luo YW, Lin YF, Yuan Y, et al. Hepatoma cell-secreted exosomal microRNA-103 increases vascular permeability and promotes metastasis by targeting junction proteins. Hepatology 2018; 68:1459-1475. 
15. Zhang X, Yang M, Lin L, Chen P, Ma KT, Zhou CY, et al. Runx2 overexpression enhances osteoblastic differentiation and mineralization in adipose-derived stem cells in vitro and in vivo. Calcif Tissue Int 2006; 79:169-178.

16. Takahashi T. Overexpression of Runx2 and MKP-1 stimulates transdifferentiation of 3T3-L1 preadipocytes into bone-forming osteoblasts in vitro. Calcif Tissue Int 2011; 88:336-347.

17. Royo F, Falcon-Perez JM. Liver extracellular vesicles in health and disease. J Extracell Vesicles 2012; 1.

18. Wesolowska A, Piwocka K. [Exosomal microRNAs as a part of the cell-cell communication in cancer]. Postepy Biochem 2017; 63:110-118.

19. Schroeder TM, Kahler RA, Li X, Westendorf JJ. Histone deacetylase 3 interacts with runx2 to repress the osteocalcin promoter and regulate osteoblast differentiation. J Biol Chem 2004; 279:4199842007.

20. Yan W, Wu X, Zhou W, Fong MY, Cao M, Liu J, et al. Cancer-cell-secreted exosomal miR-105 promotes tumour growth through the MYC-dependent metabolic reprogramming of stromal cells. Nat Cell Biol 2018; 20:597-609.

21. Zeng Z, Li Y, Pan Y, Lan X, Song F, Sun J, et al. Cancer-derived exosomal miR-25-3p promotes premetastatic niche formation by inducing vascular permeability and angiogenesis. Nat Commun 2018; 9:5395.

22. Ye Y, Li SL, Ma YY, Diao YJ, Yang L, Su MQ, et al. Exosomal miR-141-3p regulates osteoblast activity to promote the osteoblastic metastasis of prostate cancer. Oncotarget 2017; 8:94834-94849.

23. Shi P, Zhou M, Yang Y. Upregulated tumor sirtuin 2 expression correlates with reduced TNM stage and better overall survival in surgical breast cancer patients. Ir J Med Sci 2020; 189:83-89.

24. Head PE, Zhang H, Bastien AJ, Koyen AE, Withers AE, Daddacha WB, et al. Sirtuin 2 mutations in human cancers impair its function in genome maintenance. J Biol Chem 2017; 292:9919-9931.

25. Damodaran S, Damaschke N, Gawdzik J, Yang B, Shi C, Allen GO, et al. Dysregulation of Sirtuin 2 (SIRT2) and histone H3K18 acetylation pathways associates with adverse prostate cancer outcomes. BMC Cancer 2017; 17:874.

26. Hou H, Chen W, Zhao L, Zuo Q, Zhang G, Zhang X, et al. Cortactin is associated with tumour progression and poor prognosis in prostate cancer and SIRT2 other than HADC6 may work as facilitator in situ. J Clin Pathol 2012; 65:1088-1096.

27. Komori T. Regulation of Proliferation, Differentiation and Functions of Osteoblasts by Runx2. Int $J$ Mol Sci 2019; 20.

28. Adhami MD, Rashid H, Chen H, Clarke JC, Yang Y, Javed A. Loss of Runx2 in committed osteoblasts impairs postnatal skeletogenesis. J Bone Miner Res 2015; 30:71-82.

29. Otto F, Thornell AP, Crompton T, Denzel A, Gilmour KC, Rosewell IR, et al. Cbfa1, a candidate gene for cleidocranial dysplasia syndrome, is essential for osteoblast differentiation and bone development. Cell 1997; 89:765-771. 


\section{Figures}
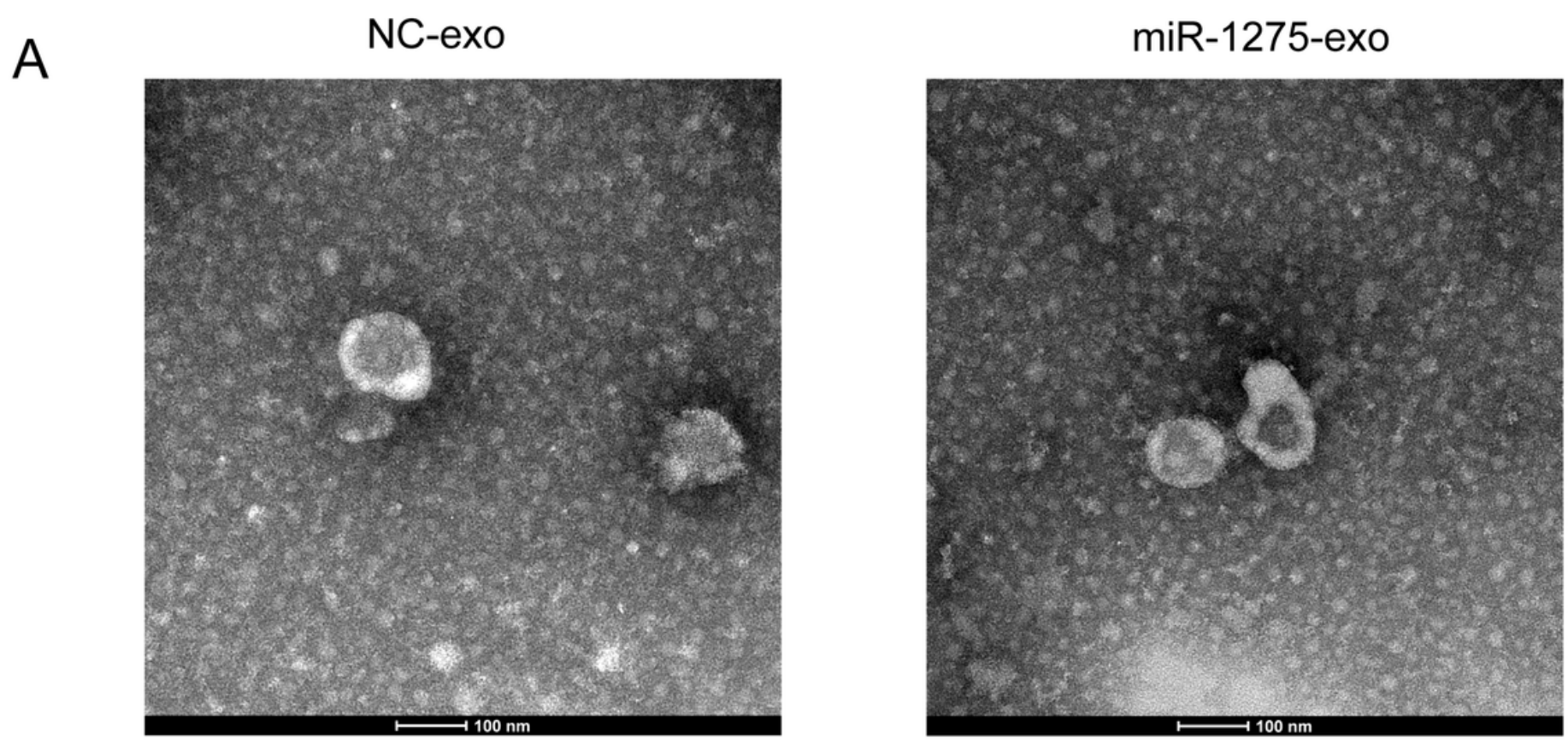

B
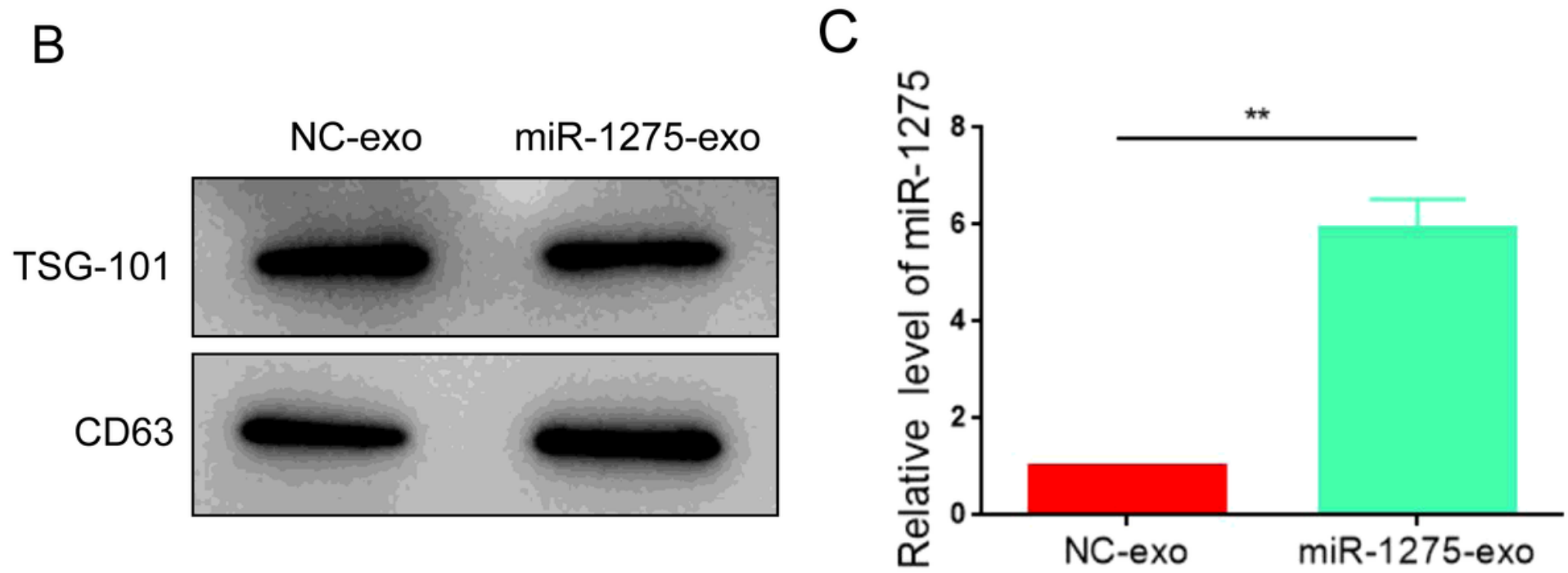

Figure 1

Characterization of exosomes from prostate cancer cells. (A) The exosome morphology was detected by transmission electron microscopy. (B) The expression of exosome markers (TSG-101 and CD63) was detected by Western blotting. (C) The expression of miR-1275 in exosomes was detected by qRT-PCR. Exosomes were extracted from prostate cancer ( $\mathrm{PCa}$ ) cells transfected with miR-1275 (miR-1275-exo) and control miRNA (NC-exo). 
A

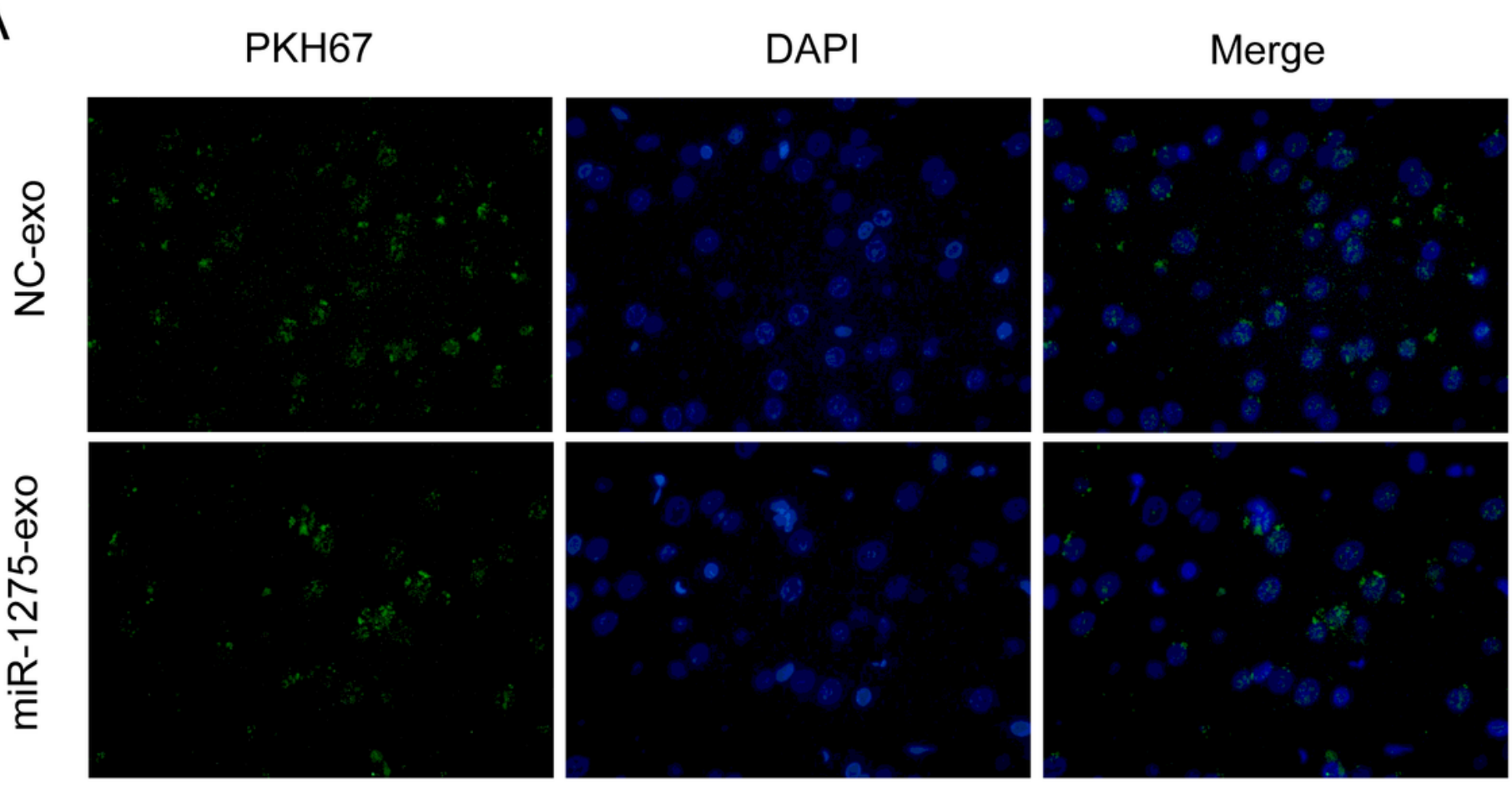

B

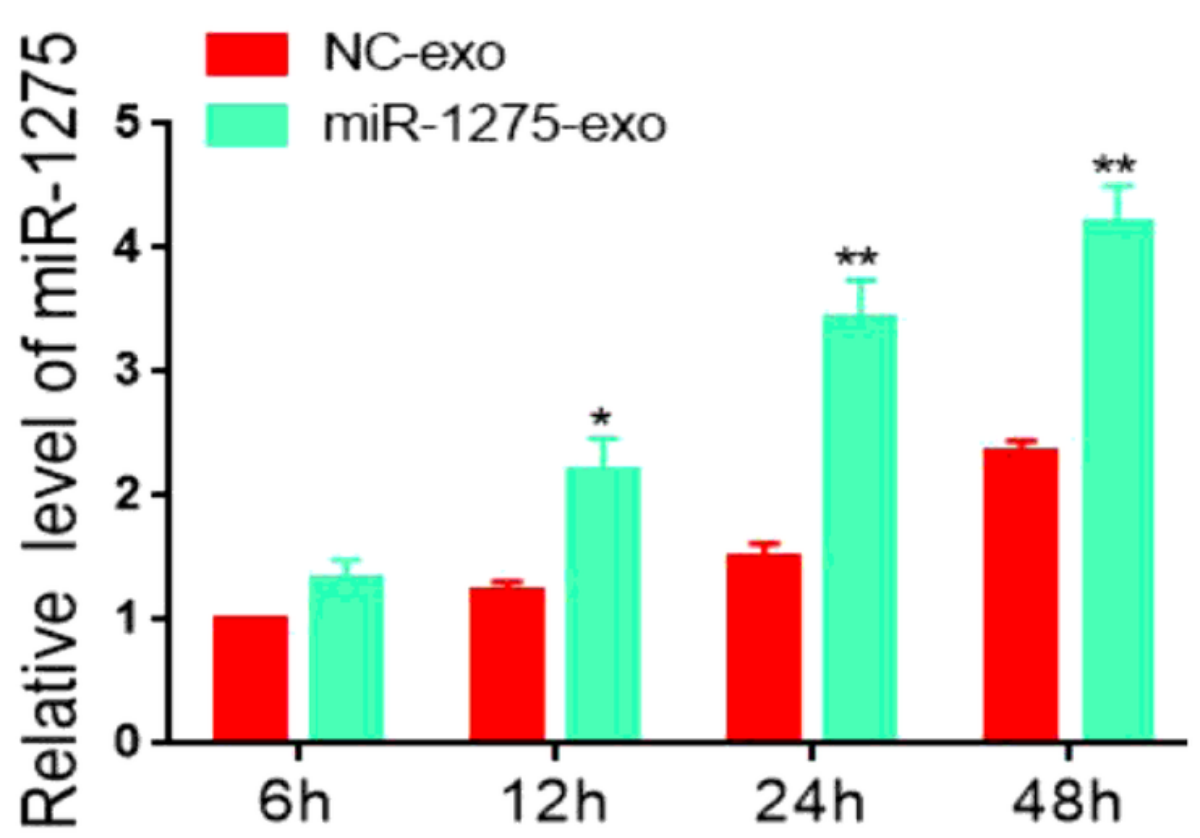

Figure 2

PCa-secreted exosomes are taken up by osteoblasts. (A) The uptake of PCa-secreted exosomes in osteoblasts was detected by immunofluorescence staining. (B) The expression of miR-1275 in osteoblasts was detected by qRT-PCR following the incubation of exosomes and osteoblasts. 
A

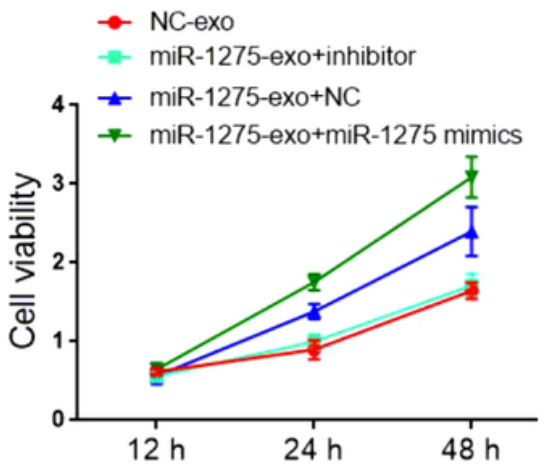

B
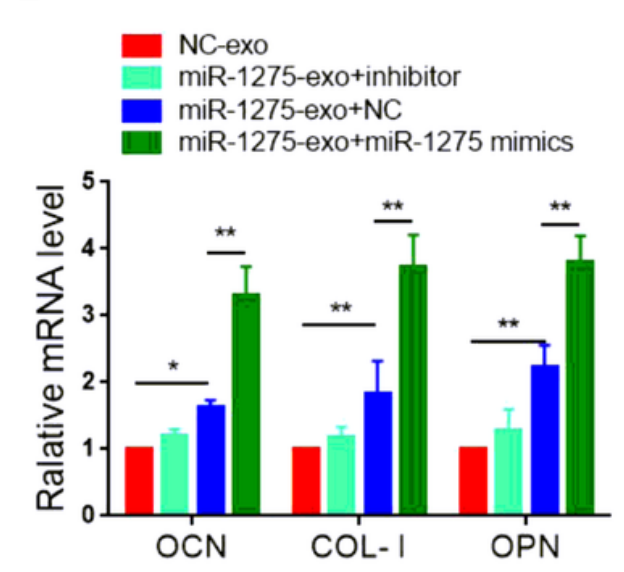

C

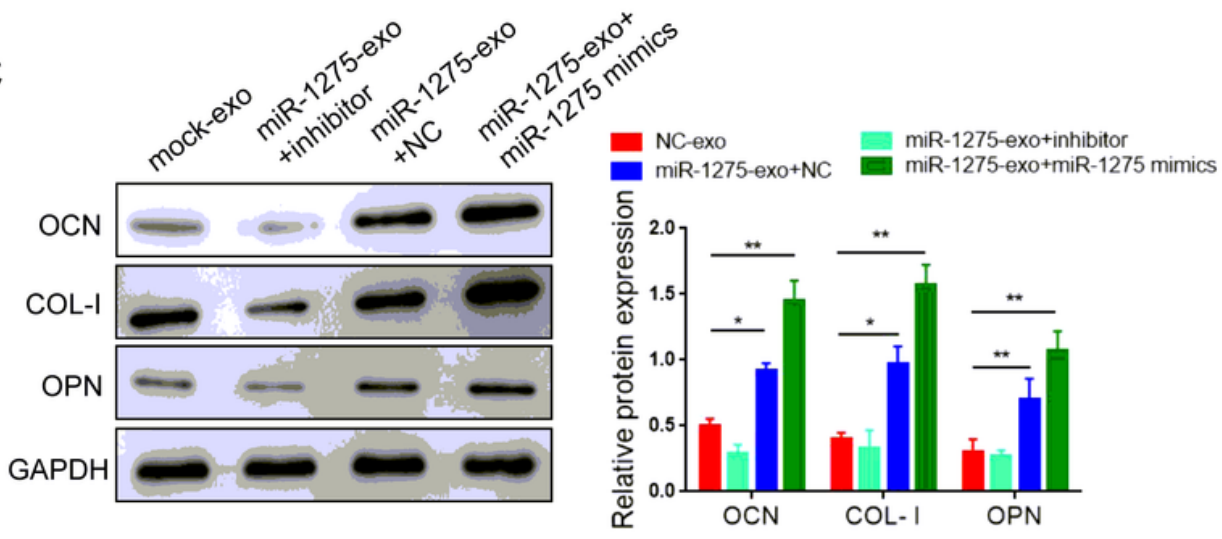

D

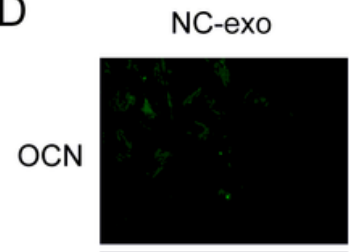

miR-1275-exo +inhibitor

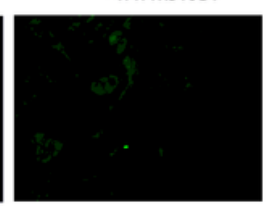

miR-1275-exo

$+\mathrm{NC}$

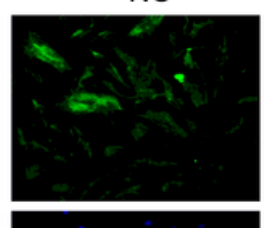

miR-1275-exo miR-1275 mimics

DAPI
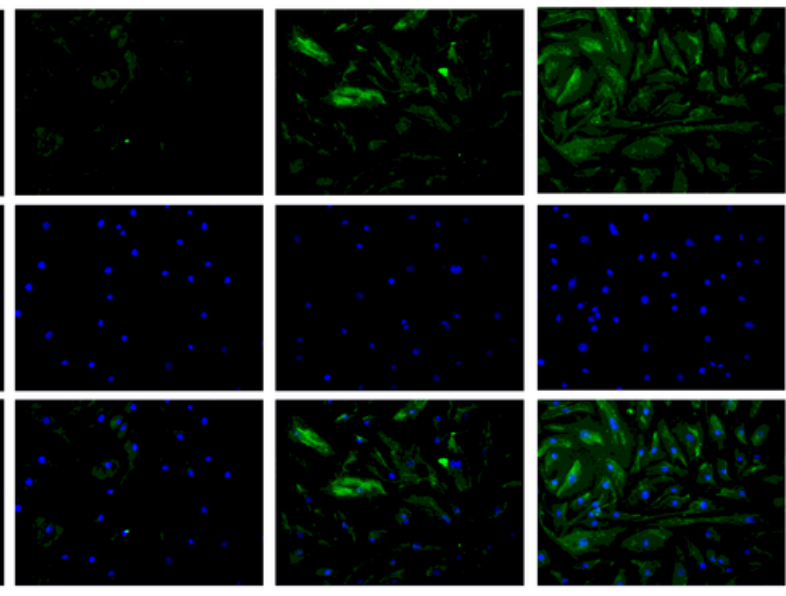

\section{Figure 3}

Exosomal miR-1275 increases osteoblast proliferation and differentiation. (A) The effect of exosomal miR-1275 on osteoblast growth was detected by CCK-8 assay. Effects of exosomal miR-1275 on the mRNA and protein expression levels of osteoblast-specific genes were determined by qRT-PCR (B), Western blot (C), and immunofluorescence staining (D), respectively. 

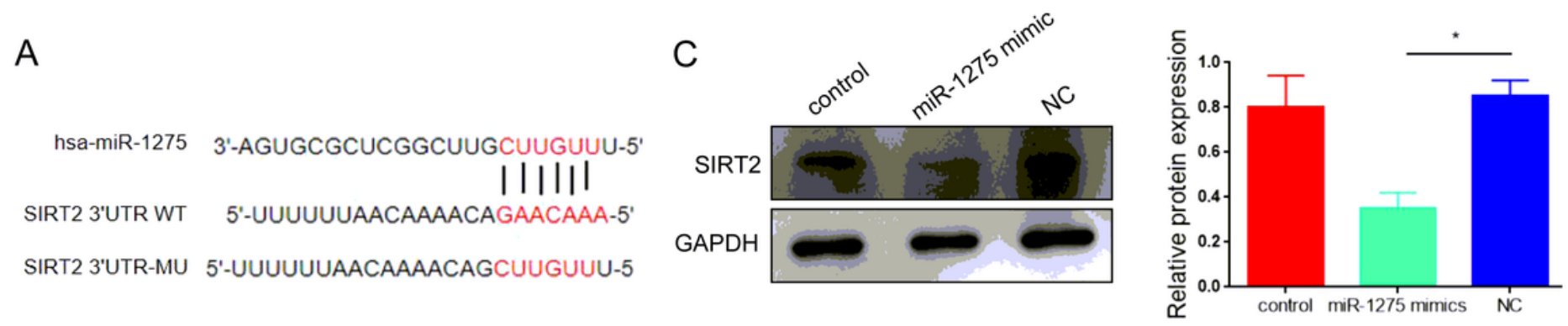

B
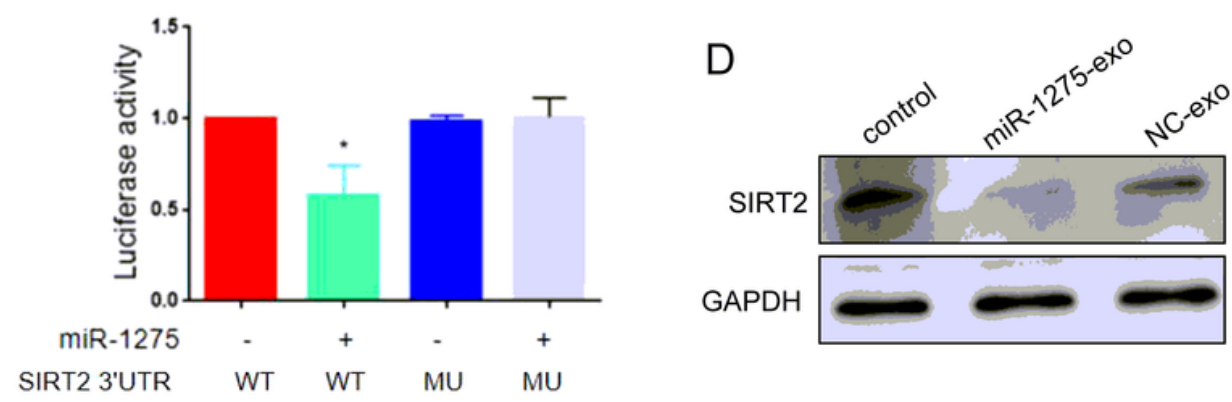

$\mathrm{E}$
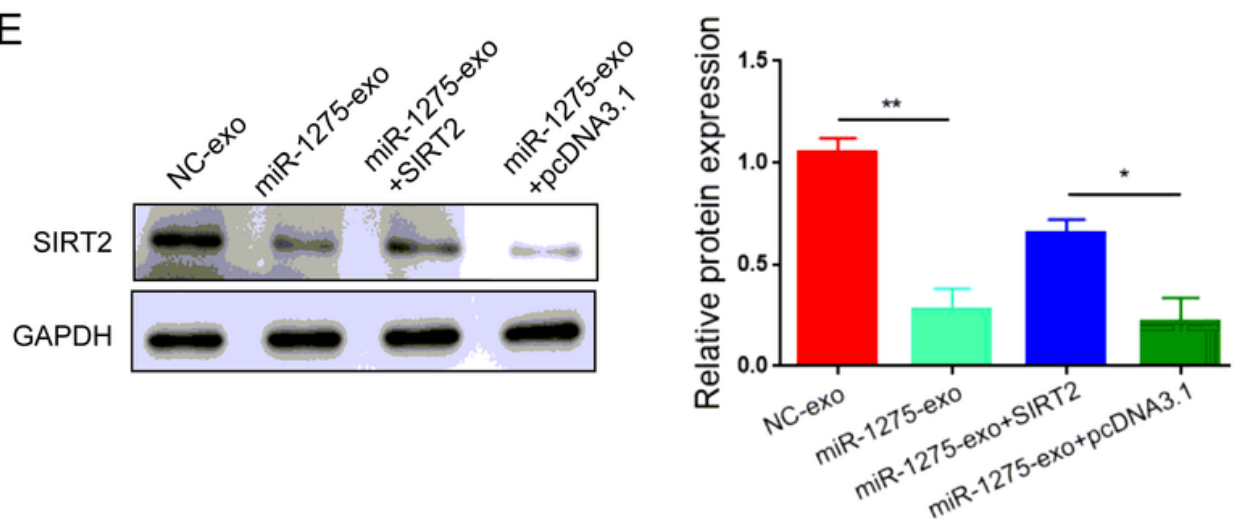

$\mathrm{F}$
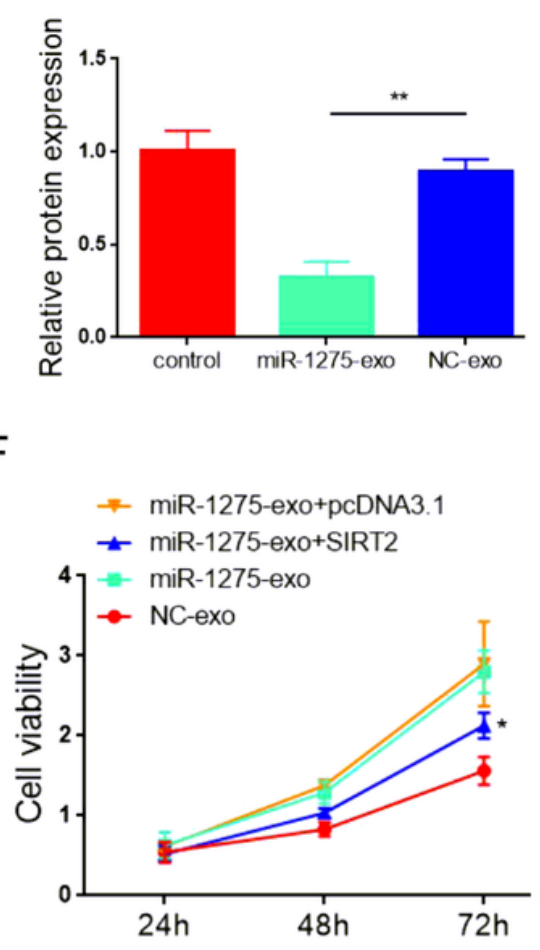

Figure 4

SIRT2 is a target of miR-1275. (A) The wild-type (WT) and mutated (Mu) binding sites of miR-1275 in the 3'UTR of SIRT2 are shown. (B) Luciferase report assay was performed in osteoblasts transfected with WT or mutated SIRT2 3'UTR in the presence (or absence) of miR-1275. (C) The effect of miR-1275 on the expression of SIRT2 was detected by Western blot. (D) The effect of exosomal miR-1275 on the expression of SIRT2 was detected by Western blot. (E) Overexpression of SIRT2 restored the expression of SIRT2 reduced by exosomal miR-1275. (F) Overexpression of SIRT2 reduced osteoblast growth increased by exosomal miR-1275. ${ }^{\star} \mathrm{P}<0.05,{ }^{\star *} \mathrm{P}<0.01$. 
A
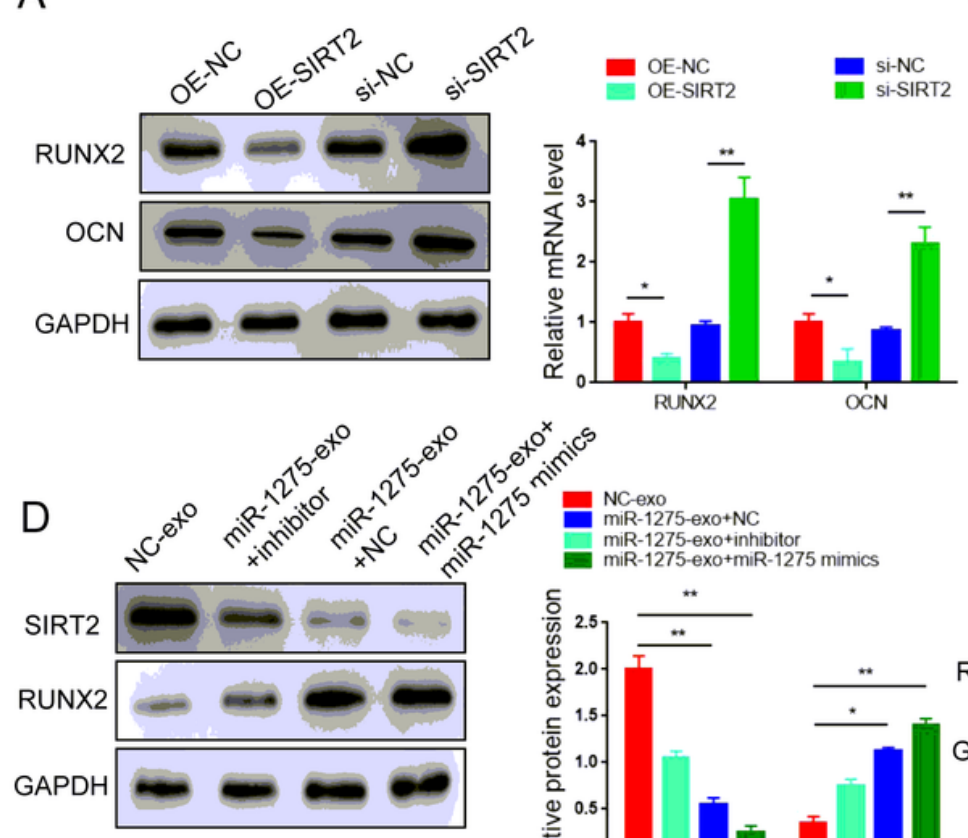

$\mathrm{F}$
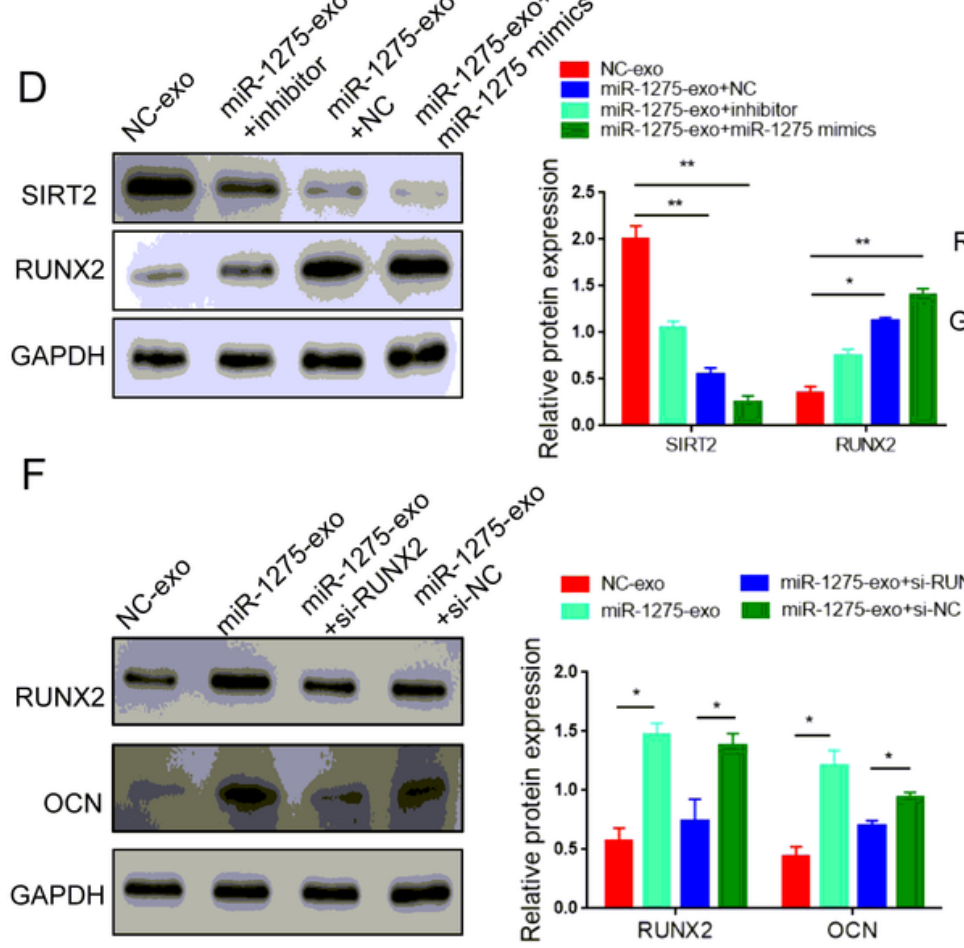

B

G

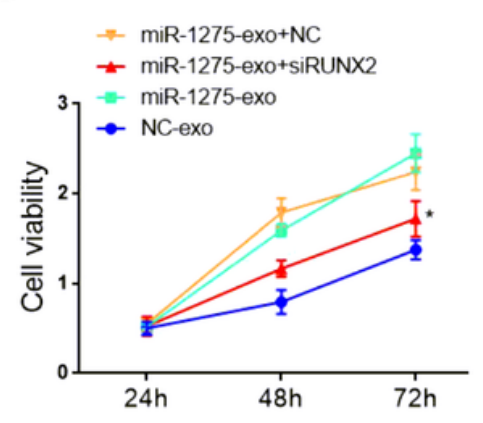

C

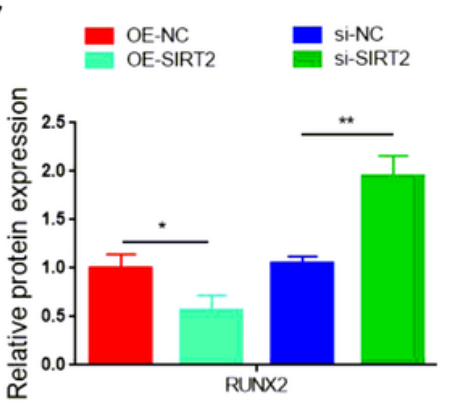

E
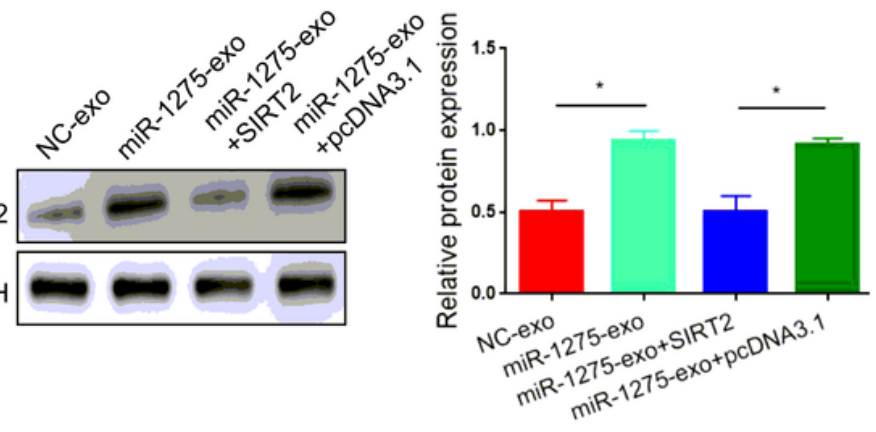

$\mathrm{H}$

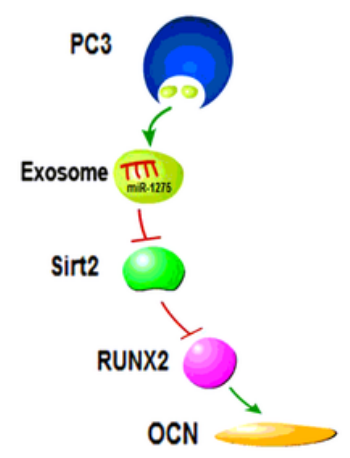

Figure 5

Exosomal miR-1275 accelerates osteoblast activity by modulating SIRT2/RUNX2 cascade. (A) The expression of RUNX2 and osteocalcin (OCN) was detected by Western blot following expression or knockdown of SIRT2. (B) The luciferase activity of RUNX2 promoter was determined in osteoblasts overexpressing SIRT2. (C) The mRNA expression of RUNX2 was detected by qRT-PCR. (D) The effect of exosomal miR-1275 on the expression of SIRT2 and RUNX2 was examined by Western blot. (E) Overexpression of SIRT2 decreased RUNX2 expression induced by exosomal miR-1275. (F) Silencing RUNX2 decreased the expression of RUNX2 increased by exosomal miR-1275. (G) Silencing RUNX2 reduced osteoblast viability mediated by exosomal miR-1275. $(\mathrm{H})$ The schematic image of the mechanism of exosomal miR-1275 promotes osteoblast activity. ${ }^{*} \mathrm{P}<0.05,{ }^{*} \mathrm{P}<0.01$. 